\title{
Identification of novel targets for the diagnosis and treatment of liver fibrosis
}

\author{
PAOLO FAGONE ${ }^{1}$, KATIA MANGANO $^{1}$, SANTA MAMMANA $^{1}$, ANTONIO PESCE ${ }^{2}$, AURORA PESCE ${ }^{2}$, \\ ROSARIO CALTABIANO ${ }^{2}$, ALEXANDRA GIORLANDINO ${ }^{2}$, TERESA ROSANNA PORTALE ${ }^{2}$, EUGENIO CAVALLI ${ }^{1}$, \\ GIUSEPPE A.G. LOMBARDO ${ }^{1}$, MARINELLA COCO ${ }^{1}$, STEFANO PULEO $^{2}$ and FERDINANDO NICOLETTI ${ }^{1}$
}

Departments of ${ }^{1}$ Biomedical Sciences and ${ }^{2}$ Medical and Surgical Sciences and

Advanced Technologies, G.F. Ingrassia, University of Catania, Catania, Italy

Received January 26, 2015; Accepted June 29, 2015

DOI: $10.3892 / \mathrm{ijmm} .2015 .2264$

\begin{abstract}
Liver fibrosis is characterized by the excessive deposition of extracellular matrix (ECM) in the hepatic parenchyma and represents an intrinsic response to chronic injury, maintaining organ integrity when extensive necrosis or apoptosis occurs. Hepatic stellate cells (HSCs) are the major cell type responsible for liver fibrosis. Following liver injury, HSCs become activated and transdifferentiate into myofibroblasts (MFBs) that lead to intrahepatic ECM accumulation. In the present study, we performed a meta-analysis of datasets which included whole-genome transcriptional data on HSCs in the quiescent and activated state from two different rodent species and identified commonly regulated genes. Several of the genes identified, including ECM components, metalloproteinases and growth factors, were found to be well-known markers for HSC activation. However, other significant genes also appeared to play important roles in hepatic fibrosis. The elucidation of the molecular events underlying HSC activation may be key to the identification of potential novel pharmacological targets for the prevention and treatment of liver fibrosis.
\end{abstract}

\section{Introduction}

Liver fibrosis is characterized by the excessive deposition of extracellular matrix (ECM) in the hepatic parenchyma and occurs following complex interactions between matrix-producing hepatic stellate cells (HSCs) and liver-resident and infiltrating cells (1). Fibrosis is an intrinsic response to chronic injury, maintaining organ integrity when extensive necrosis or apoptosis occurs. With protracted damage, fibrosis can progress toward excessive scarring and organ failure, as

Correspondence to: Professor Ferdinando Nicoletti, Department of Biomedical Sciences, University of Catania, Via Androne 83, I-95124 Catania (CT), Italy

E-mail: ferdinic@unict.it

Key words: liver, hepatic fibrosis, hepatic stellate cells, myofibroblast, bioinformatics in liver cirrhosis. Although hepatic fibrosis in humans can be caused by various stimuli (congenital, metabolic, inflammatory, parasitic, vascular, toxins or drugs), the molecular mechanisms underlying the development of fibrosis are basically the same (2). Following liver injury, a defined program of molecular changes occurs that is tightly orchestrated at the cellular and molecular level (3). Importantly, fibrosis is no longer considered static, but the result of a continuous remodeling process. This process is characterized mainly by the activation of HSCs which acquire a myofibroblast (MFB) phenotype and are able to express and deposit large quantities of ECM components within the liver $(4,5)$. If the injury is temporary, these changes are transient and liver fibrosis may not occur. If the injury is sustained, however, chronic inflammation and the accumulation of the ECM persist, leading to the progressive substitution of the normal liver parenchyma by scar tissue. In the pathogenesis of chronic liver disease, the ECM homeostasis is further disturbed by an unbalanced activity of matrix metalloproteinases (MMPs) and their tissue inhibitors (TIMPs). Experimental studies conducted on isolated primary hepatic cells and experimental animal models have led to the identification of several pathogenetic mediators, namely signaling pathways that are involved in the fibrogenic response. The aberrant activity of transforming growth factor (TGF)- $\beta 1$ or members of the platelet-derived growth factor family are the most prominent drivers of the activation and transdifferentiation of HSCs into MFBs.

To date, the antifibrotic treatment of fibrosis represents an unconquered area for drug development, with enormous potential but also high risks. Preclinical research has yielded numerous targets for antifibrotic agents, some of which have entered early-phase clinical studies, but progress has been hampered due to the relative lack of sensitive and specific biomarkers to measure the progression or reversal of fibrosis $(6,7)$. The aim of the present study was to identify candidate genes and putative biomarkers for the diagnosis and treatment of hepatic fibrosis.

\section{Materials and methods}

Initial screening of microarray datasets. The microarray datasets used in this study were obtained from NCBI Gene Expression Omnibus (GEO, http://www.ncbi.nlm.nih. 


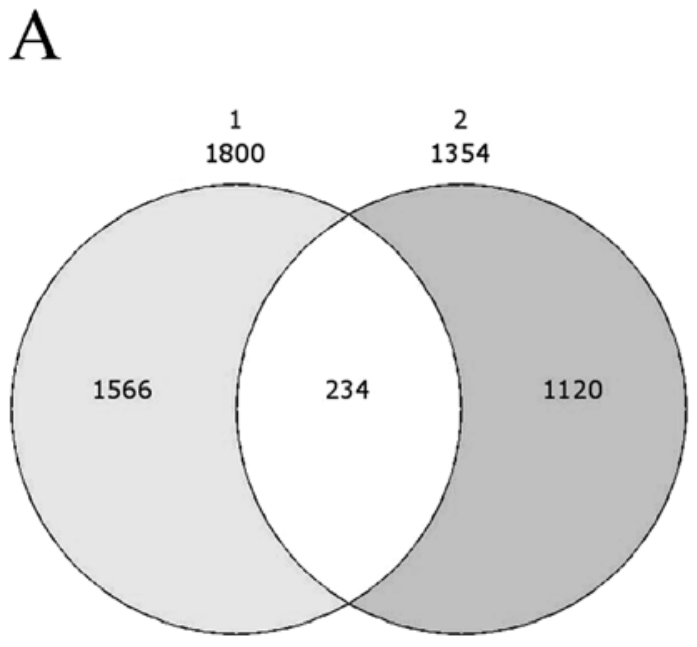

Lists contain 2920 unique elements

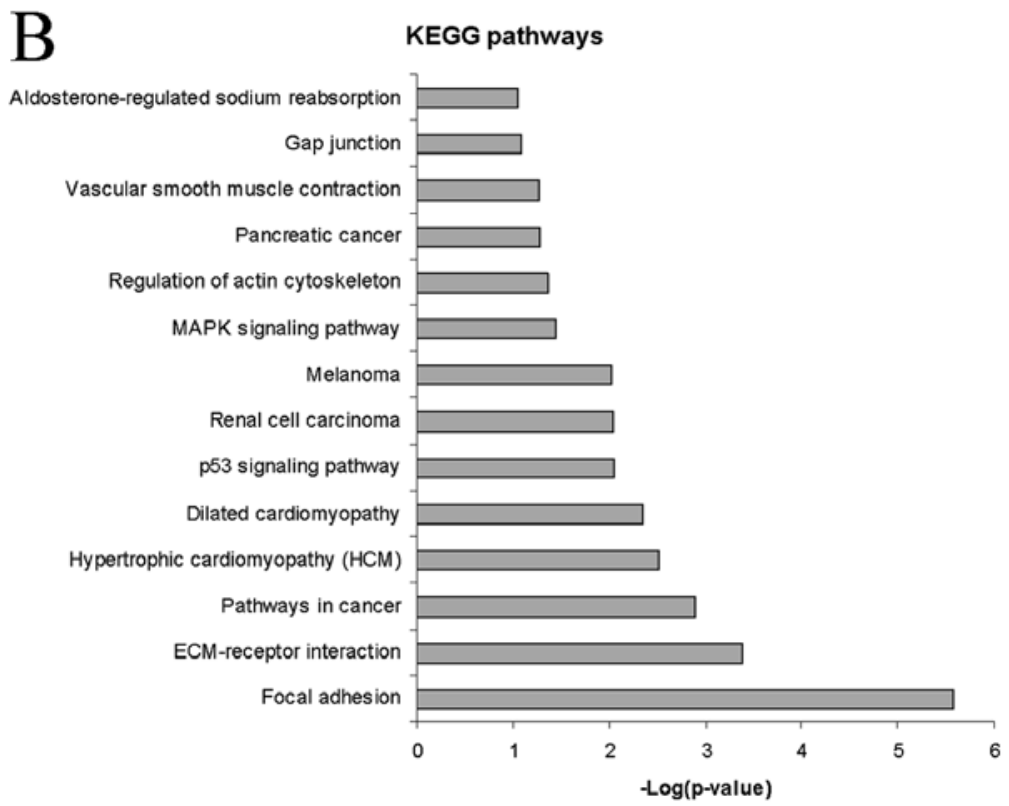

Figure 1. Definition of a fibrogenic gene signature. (A) Venn diagram showing common upregulated genes in the GSE34949 and E-MTAB-2081 datasets. (B) Most enriched KEGG pathways among the commonly upregulated genes.

gov/geo/) $(8,9)$ and ArrayExpress (10). MESH terms 'liver fibrosis', 'myofibroblasts' and 'hepatic stellate cells' were used to identify potential datasets of interest, and GSE34949 and E-MTAB-2081 were selected for the analysis. Both datasets included whole-genome transcriptional data from rodent HSCs in the quiescent and activated state. For GSE34949, total RNA was extracted from primary C57BL/6 mouse HSCs on days 0 and 7 of culture. For the E-MTAB-2081 dataset, RNA samples were obtained from quiescent and activated HSCs derived from Sprague-Dawley rats. For each dataset, we performed moderate $\mathrm{t}$-statistics to generate a list of upregulated genes. We used a threshold value of $p<0.05$ and a fold change of $>2$. In order to identify a robust fibrogenic gene signature, the web-based application, Venn Diagram Generator (http:/www.bioinformatics. lu/venn.php), was used to select the differentially expressed genes (DEGs) commonly shared between the two datasets.

Functional analysis of genes of the fibrogenic gene signature. Functional analysis was performed on the selected genes using the Database for Annotation, Visualization and Integrated Discovery (DAVID) Bioinformatics Database (http://david. abcc.ncifcrf.gov/), as previously described (11). The biological significance of these genes was queried by performing enrichment analysis for Gene Ontology (GO) categories, including biological processes (BPs) and molecular functions (MFs), for domain architectures [Simple Modular Architecture Research Tool (SMART)] and for the identification of significant biological pathways. The Kyoto Encyclopedia of Genes and Genomes (KEGG), a collection of manually curated pathways and molecular networks, was used as reference. In order to construct a series of interconnected networks, the selected genes were subjected to k-means clustering using the STRING database (http://string-db.org/). STRING provides a global view of all the available interaction data by creating networks, which represent the current knowledge on the functional interconnections among genes. All associations are provided with a confidence score that represents an estimation on how likely a given association describes a functional linkage between two genes. Clustering was performed for five clusters and disconnected nodes were excluded. The web server, REVIGO (http://revigo.irb.hr), was used to summarize the lists of GO terms in order to find representative subsets of the terms that rely on semantic similarity.

\section{Results}

Identification of fibrogenic genes. We identified 1,800 genes which were upregulated in the activated vs. the resting HSCs in the GSE34949 dataset and 1,354 genes in the E-MTAB-2081 dataset. Among the significantly upregulated genes, 254 genes were common between the two datasets and were selected for further analysis (Fig. 1A). As expected, some genes had well-established associations with fibrosis. These included ECM components [collagen, type I, alpha 1 (COL1A1), collagen, type I, alpha 2 (COL1A2), collagen, type III, alpha 1 (COL3A1), collagen, type $\mathrm{V}$, alpha 2 (COL5A2), collagen, type VIII, alpha 1 (COL8A1), collagen, type XII, alpha 1 (COL12A1), fibronectin 1 (FN1) and tenascin C (TNC)], metalloproteinases (MMP11, MMP12, MMP19 and MMP23) and growth factors [TGFB2, TGFB3, fibroblast growth factor (FGF)18 and FGF2].

Functional analysis of commonly upregulated genes. We utilized DAVID to identify the major functions, networks and pathways relevant to the 254 significantly upregulated genes.

Pathway analysis revealed that the top three significant pathways enriched by the 254 genes were 'focal adhesion' (Figs. 1B and 3), 'ECM-receptor interaction' and 'pathways in cancer'. These pathways shared several genes, i.e., the FGFs and ECM components already indicated. Other notable genes were represented by protein kinase $\mathrm{C}$, alpha (PRKCA), insulin-like growth factor (IGF1), filamin C, gamma (FLNC), myosin, light 

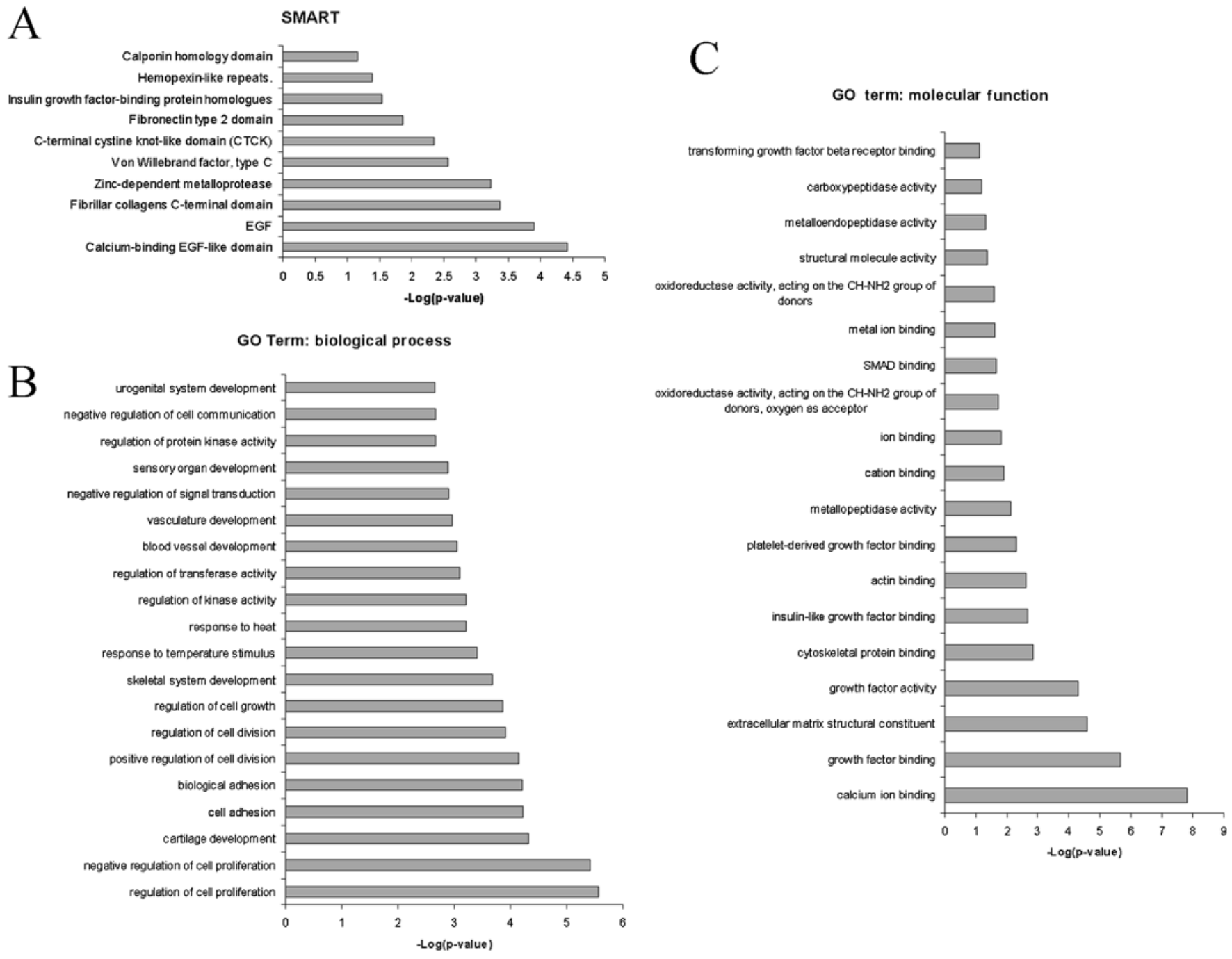

Figure 2. Functional analysis of genes of the fibrogenic gene signature. (A) Most enriched Simple Modular Architecture Research Tool (SMART) categories enriched by the commonly upregulated genes between the GSE34949 and E-MTAB-2081 datasets. Most represented (B) biological processes (BPs) and (C) molecular functions (MFs) among the commonly upregulated genes between the GSE34949 and E-MTAB-2081 datasets.

chain 9, regulatory (MYL9), p21 protein (Cdc42/Rac)-activated kinase 3 (PAK3), platelet derived growth factor C (PDGFC) and c-fos-induced growth factor/vascular endothelial growth factor D (FIGF) in the 'focal adhesion' pathway, syndecan 2 (SDC2) in the 'ECM-receptor interaction' pathway and PRKCA, cyclin-dependent kinase inhibitor 2A (CDKN2A), cyclin-dependent kinase inhibitor 2B (CDKN2B), arylhydrocarbon receptor nuclear translocator 2 (ARNT2), IGF1, frizzled class receptor 2 (FZD2) and FIGF in the "pathways in cancer'. Among the other top enriched KEGG pathways, the presence of those genes related to hypertrofic and dilated cardiomyopathy was indicative of the myofibroblastic transdifferentiation of activated HSCs (Fig. 1B). In addition, the involvement of the p53, renal cell carcinoma, melanoma and mitogen-activated protein kinase (MAPK) signaling pathways seemed to correlate with liver fibrosis to liver cirrhosis and the evolution toward hepatocellular carcinoma (Fig. 1B), and as previously demonstrated (12).

The use of SMART led to the identification of the 'calcium-binding EGF-like domain', 'EGF', 'fibrillar collagens
C-terminal domain', 'zinc-dependent metalloprotease' and 'von Willebrand factor, type $\mathrm{C}$ ' as the principal protein domains overrepresented by the commonly upregulated genes during fibrogenesis (Fig. 2A and B).

A characteristic of HSCs is the ability to undergo cell cycle progression and cell mitosis $(13,14)$. Indeed, biological processes strongly associated with the upregulated genes were found to be related to the regulation of cell proliferation, cartilage development, regulation of cell growth and division (Fig. 2B).

The most significant molecular functions (Fig. 2C) associated with the commonly upregulated genes were 'calcium ion binding', 'growth factor binding', 'extracellular matrix structural constituent' and 'growth factor activity' (Fig. 2C).

Gene network analysis of upregulated genes. The sole analysis of the gene expression profile provides an incomplete picture of the biological mechanisms involved in a process, such as liver fibrosis, since it does not include the interrelations and communication paths among genes. The construction of a gene 


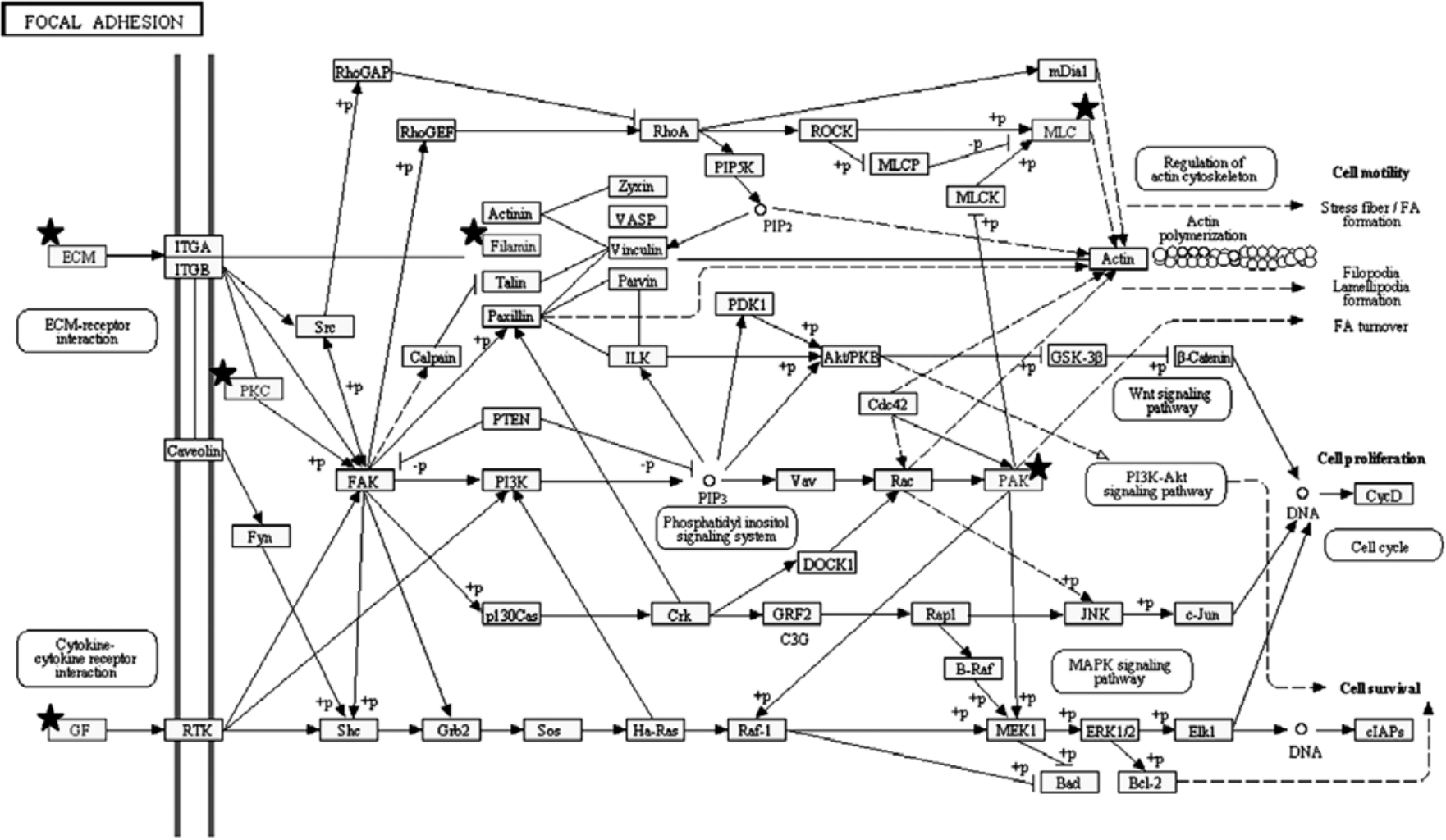

Figure 3. KEGG pathway analysis of the commonly upregulated genes between the GSE34949 and E-MTAB-2081 datasets indicated 'focal adhesion' as the most enriched pathway. Genes commonly upregulated between the GSE34949 and E-MTAB-2081 datasets are highlighted using star symbols.

network is the logical continuation of gene expression profiling. Describing a biological system using a network helps to identify characteristics that could potentially lead to the development of novel treatment strategies, by a better understanding of the process. In a gene network, genes form the network nodes and relationships serve as network edges.

Genes were clustered using the k-means algorithm for a number of five clusters (Fig. 4). The central hub was occupied by FN1 which interconnected all of the five clusters. Other important nodes were represented by caldesmon 1 (CALD1), COL1A1 and FGF2.

CALD1 is a protein expressed in smooth muscle cells, which combines with calmodulin, tropomyosin and actin thus regulates cellular contraction (15). Although it has been suggested to be a biomarker for smooth muscle tumors, its role in liver fibrosis remains unexplored.

Another important gene, at the interface of three different clusters, was represented by S100A4. S100A4, also known as fibroblast-specific protein 1 (FSP1), belongs to the S100 gene family, a multi-gene family of highly conserved $\mathrm{Ca}^{2+}$-binding proteins. S100A4 is known to be upregulated in pulmonary fibrosis (16), renal fibrosis (17) and in portal tract fibrosis following bile duct damage (18). Data on endometrial cancer have indicasted that S100A4 is induced by TGF- $\beta 1$ and that it is necessary for the effects of TGF- $\beta 1$ on cancer cell migration and invasion (19). S100A4 could thus play a central role in the induction and/or maintenance of liver fibrosis in chronic hepatic disease. The development of drugs specifically targeting S100A4 may thus be equally effective as anti-TGF- $\beta$ treatment, but with less potential adverse effects, given the more downstream target.
Another gene that was identified as a relevant hub in the network analysis was Runt-related transcription factor 2 (Runx2). The Runx family of transcription factors is composed of three distinct genes, Runx1, Runx 2 and Runx3, which play a key part in normal development and cancer (20). In particular, Runx 2 is involved in osteoblast differentiation and bone formation and it has been reported that Runx 2 is a downstream target of the TGF/Smad4 pathway. In addition, the MAPK pathway plays an essential role in the phosphorylation and activation of Runx2 (21). Hattori et al (22) demonstrated that the inhibition of p38 is able to ameliorate liver cirrhosis through the downregulation of Runx2. Taken together, these data suggest the central involvement of this gene in the progression of hepatic fibrosis.

Analysis of downregulated genes. In order to define the complete mechanisms underlying the development of liver fibrosis, we identified commonly downregulated genes between the GSE34949 and E-MTAB-2081 datasets. We found 1,200 and 1,122 genes downregulated in these databases, respectively, with 132 genes being shared between the two datasets. GO analysis revealed five major biological processes enriched by these genes, represented by 'response to wounding', 'positive regulation of developmental process', 'coagulation', 'carboxilic acid catabolism' and 'polysaccharide metabolism'. Fig. 5 shows a scatterplot of the cluster representatives obtained by applying multidimensional scaling to a matrix of the semantic similarities of the GO terms. Along the same lines, KEGG pathway analysis identified as the most enriched pathways, the complement and coagulation 


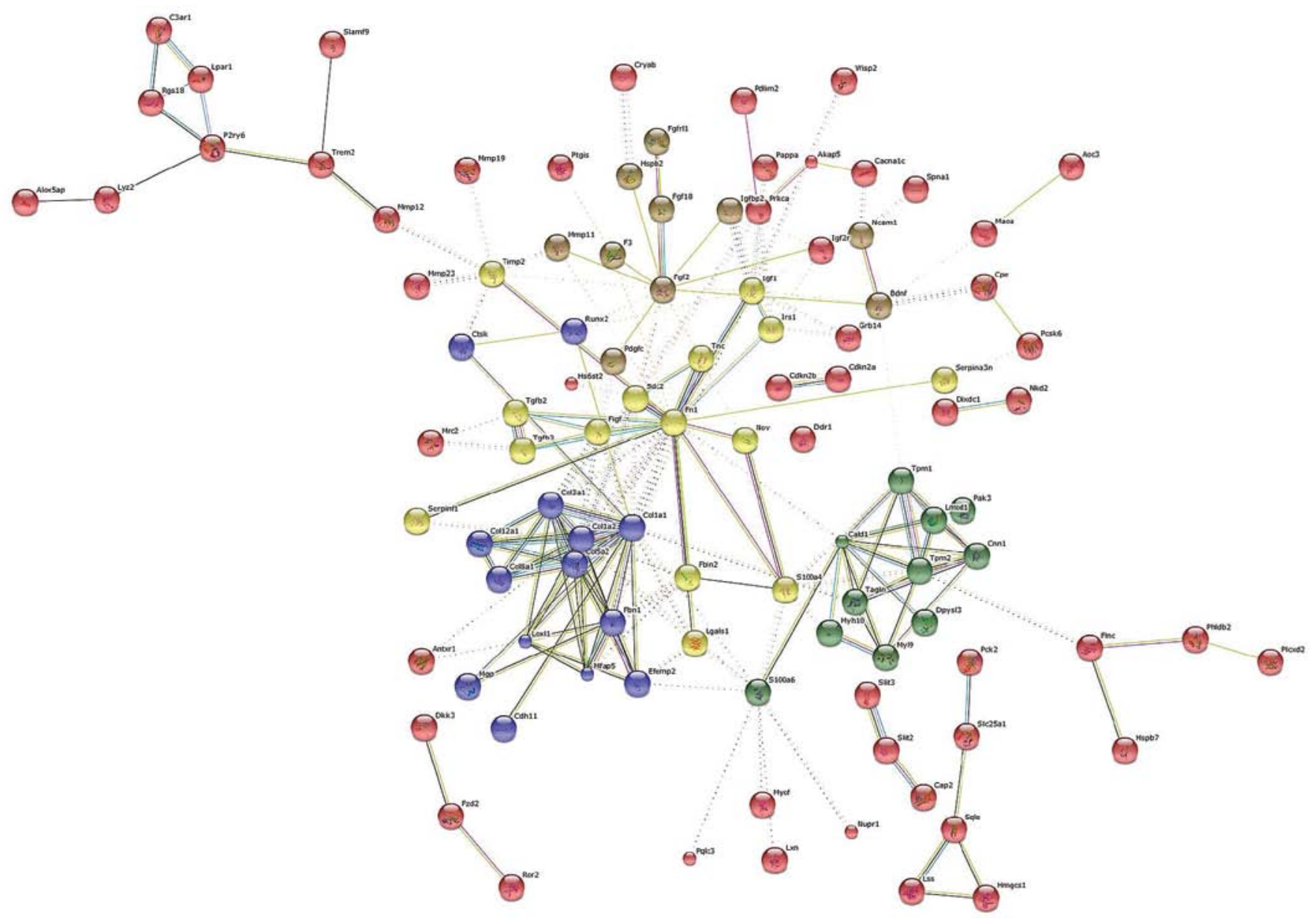

Figure 4. Fibrogenic gene network. Gene network was constructed using the commonly upregulated genes between the GSE34949 and E-MTAB-2081 datasets. Genes were subjected to k-means clustering using the STRING database. Clustering was performed for five clusters and disconnected nodes were excluded.

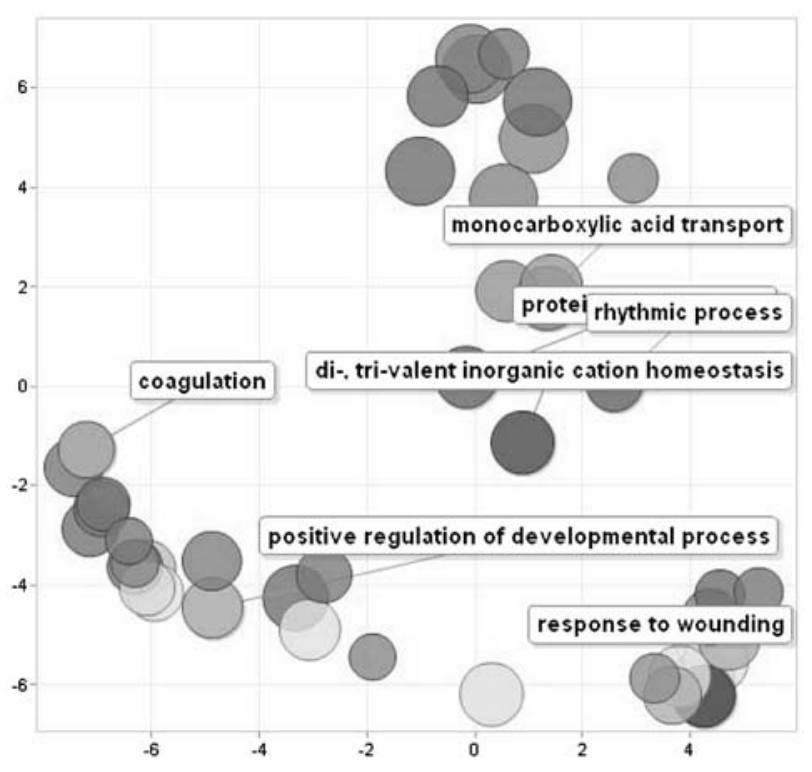

Figure 5. Gene ontology (GO) analysis of downregulated genes. Scatterplot of the cluster GO term representatives for biological processes (BPs) enriched among the commonly downregulated genes between the GSE34949 and E-MTAB-2081 datasets.

cascades' [with kininogen 1 (KNG1), fibrinogen gamma chain (FGG), fibrinogen alpha chain (FGA), fibrinogen beta chain
(FGB), coagulation factor II (thrombin) (F2), serpin peptidase inhibitor, clade C (antithrombin), member 1 (SERPINC1) and coagulation factor IX (F9) being involved], the 'NOD-like receptor signaling pathway' [which included MAPK12, interleukin (IL)1B, nuclear factor of kappa light polypeptide gene enhancer in B-cells inhibitor, alpha (NFKBIA) and MAPK11], 'cytokine-cytokine receptor interaction' [including pro-platelet basic protein (chemokine (C-X-C motif) ligand 7) (PPBP), IL1B, nerve growth factor receptor (NGFR), interleukin 13 receptor, alpha 1 (IL13RA1), IL10, IL1A and kinase insert domain receptor (KDR)] and the 'RIG-I-like receptor signaling pathway' [with MAPK12, NFKBIA, MAPK11 and DEXH (Asp-Glu-X-His) box polypeptide 58 (DHX58)]. These data are concordant with those presented in the study by Ji et al (23) who found that downregulated genes were primarily related to immune response and lipid metabolism. Of note, GO analysis revealed that the major molecular functions involved were the endopeptidase inhibitor, the vitamin transporter, protein bridging and DNA-directed DNA polymerase activity.

\section{Discussion}

HSCs represent the major cell type responsible for the development of liver fibrosis. Following liver injury, HSCs become activated and transdifferentiate into MFBs that lead to intrahepatic ECM accumulation. Depicting the molecular events 
that occur during HSC activation is key for a comprehensive understanding of the biological role of HSCs in liver fibrosis and for the development of effective strategies for the diagnosis and treatment of this disorder.

Meta-analysis of multiple microarray datasets yields more reliable and comprehensive results than using a single dataset of gene expression profiles. Moreover, the possibility of including data from different mammal species increases even more the predictability power of the data extracted from a single dataset (24).

In the present study, we performed a meta-analysis of two datasets from two different rodent species and identified 254 and 132 genes that were significantly upregulated and downregulated in each dataset, respectively, in activated HSCs. Together these genes draw a robust and reliable picture of the molecular mechanisms involved in the development of hepatic fibrosis. The majority of the genes identified in this meta-analysis corresponded to factors already described in the literature by independent studies $(3,5,14,18,23)$. This observation strongly supports the reliability of the data presented herein. These data allow researchers to identify novel pharmacological targets to prevent and treat hepatic fibrogenesis, and to monitor its evolution through the screening of relevant molecules involved in the degeneration of the liver parenchyma. The ideal biomarker for liver fibrosis should be reliable and readily available and should be useful in assessing the progression of liver disease. The identification of a biomarker with such characteristics would replace the need for biopsies which, being invasive procedures, are associated with the risk of pain and/or bleeding.

Among the list of candidate genes identified in this study, there are several highly modulated genes which have not been previously associated with liver fibrosis. Bioinformatics analysis of these modulated genes not only enhanced our understanding of the major characteristics of activated HSCs, but also suggested new avenues for the development of more effective therapies for liver fibrosis.

\section{References}

1. Anthony PP, Ishak KG, Nayak NC, Poulsen HE, Scheuer PJ and Sobin LH: The morphology of cirrhosis. Recommendations on definition, nomenclature, and classification by a working group sponsored by the World Health Organization. J Clin Pathol 31: 395-414, 1978

2. Hernandez-Gea V and Friedman SL: Pathogenesis of liver fibrosis. Annu Rev Pathol 6: 425-456, 2011.

3. Gressner AM and Weiskirchen R: Modern pathogenetic concepts of liver fibrosis suggest stellate cells and TGF- $\beta$ as major players and therapeutic targets. J Cell Mol Med 10: 76-99, 2006.

4. Friedman SL: Hepatic stellate cells: Protean, multifunctional, and enigmatic cells of the liver. Physiol Rev 88: 125-172, 2008.

5. Tacke $\mathrm{F}$ and Weiskirchen R: Update on hepatic stellate cells: Pathogenic role in liver fibrosis and novel isolation techniques. Expert Rev Gastroenterol Hepatol 6: 67-80, 2012.

6. Popov Y and Schuppan D: Targeting liver fibrosis: strategies for development and validation of antifibrotic therapies. Hepatology 50: 1294-1306, 2009.
7. Schuppan D and Kim YO: Evolving therapies for liver fibrosis. J Clin Invest 123: 1887-1901, 2013.

8. Nicoletti A, Fagone P, Donzuso G, Mangano K, Dibilio V, Caponnetto S, Bendtzen K, Zappia M and Nicoletti F: Parkinson's disease is associated with increased serum levels of macrophage migration inhibitory factor. Cytokine 55: 165-167, 2011.

9. Fagone P, Di Rosa M, Palumbo M, De Gregorio C, Nicoletti F and Malaguarnera L: Modulation of heat shock proteins during macrophage differentiation. Inflamm Res 61: 1131-1139, 2012.

10. Parkinson H, Sarkans U, Kolesnikov N, Abeygunawardena N, Burdett T, Dylag M, Emam I, Farne A, Hastings E, Holloway E, et al: ArrayExpress update - an archive of microarray and high-throughput sequencing-based functional genomics experiments. Nucleic Acids Res 39 (Database issue): D1002-D1004, 2011.

11. Fagone P, Muthumani K, Mangano K, Magro G, Meroni PL, Kim JJ, Sardesai NY, Weiner DB and Nicoletti F: VGX-1027 modulates genes involved in lipopolysaccharide-induced Toll-like receptor 4 activation and in a murine model of systemic lupus erythematosus. Immunology 142: 594-602, 2014.

12. Amann T, Bataille F, Spruss T, Mühlbauer M, Gäbele E, Schölmerich J, Kiefer P, Bosserhoff AK and Hellerbrand C: Activated hepatic stellate cells promote tumorigenicity of hepatocellular carcinoma. Cancer Sci 100: 646-653, 2009.

13. Bissell DM, Wang SS, Jarnagin WR and Roll FJ: Cell-specific expression of transforming growth factor-beta in rat liver. Evidence for autocrine regulation of hepatocyte proliferation. J Clin Invest 96: 447-455, 1995.

14. Coker RK, Laurent GJ, Shahzeidi S, Lympany PA, du Bois RM, Jeffery PK and McAnulty RJ: Transforming growth factorsbeta 1 , -beta 2 , and -beta 3 stimulate fibroblast procollagen production in vitro but are differentially expressed during bleomycin-induced lung fibrosis. Am J Pathol 150: 981-991, 1997.

15. Watanabe K, Tajino T, Sekiguchi M and Suzuki T: h-Caldesmon as a specific marker for smooth muscle tumors. Comparison with other smooth muscle markers in bone tumors. Am J Clin Pathol 113: 663-668, 2000.

16. Lawson WE, Polosukhin VV, Zoia O, Stathopoulos GT, Han W, Plieth D, Loyd JE, Neilson EG and Blackwell TS: Characterization of fibroblast-specific protein 1 in pulmonary fibrosis. Am J Respir Crit Care Med 171: 899-907, 2005.

17. Robertson H, Ali S, McDonnell BJ, Burt AD and Kirby JA: Chronic renal allograft dysfunction: The role of $\mathrm{T}$ cell-mediated tubular epithelial to mesenchymal cell transition. J Am Soc Nephrol 15: 390-397, 2004.

18. Rygiel KA, Robertson H, Marshall HL, Pekalski M, Zhao L, Booth TA, Jones DE, Burt AD and Kirby JA: Epithelial-mesenchymal transition contributes to portal tract fibrogenesis during human chronic liver disease. Lab Invest 88: 112-123, 2008.

19. Xie R, Schlumbrecht MP, Shipley GL, Xie S, Bassett RL Jr and Broaddus RR: S100A4 mediates endometrial cancer invasion and is a target of TGF-beta1 signaling. Lab Invest 89: 937-947, 2009.

20. Ito Y: Oncogenic potential of the RUNX gene family: 'overview'. Oncogene 23: 4198-4208, 2004.

21. Xiao G, Jiang D, Thomas P, Benson MD, Guan K, Karsenty G and Franceschi RT: MAPK pathways activate and phosphorylate the osteoblast-specific transcription factor, Cbfa1. J Biol Chem 275: 4453-4459, 2000.

22. Hattori S, Dhar DK, Hara N, Tonomoto Y, Onoda T, Ono T, Yamanoi A, Tachibana M, Tsuchiya M and Nagasue N: FR-167653, a selective p38 MAPK inhibitor, exerts salutary effect on liver cirrhosis through downregulation of Runx2. Lab Invest 87: 591-601, 2007.

23. Ji J, Yu F, Ji Q, Li Z, Wang K, Zhang J, Lu J, Chen L, E Q, Zeng Y and Ji Y: Comparative proteomic analysis of rat hepatic stellate cell activation: A comprehensive view and suppressed immune response. Hepatology 56: 332-349, 2012.

24. Rasche A, Al-Hasani H and Herwig R: Meta-analysis approach identifies candidate genes and associated molecular networks for type-2 diabetes mellitus. BMC Genomics 9: 310, 2008. 